\title{
Effects of Varying Levels of Fly Ash and Vermicompost Amendment on Floristic Composition of Weeds in Rice Nursery
}

\author{
Sanat Kumar Dwibedi ${ }^{1}$, Sanjat Kumar Sahu ${ }^{2}$, Ravi Kumar Patnaik ${ }^{3}$, \\ Ranjan Kumar Tarai ${ }^{4}$ and Abhiram Dash ${ }^{5}$ \\ ${ }^{1}$ (Agronomy), ${ }^{4}$ (Horticulture), ${ }^{5}$ (Agricultural Statistics), Orissa University of Agriculture and \\ Technology, Bhubaneswar, Odisha, India \\ ${ }^{2}$ Department of Environmental Sciences, Jyoti Vihar, Sambalpur, Odisha, India \\ ${ }^{3}$ Orissa University of Agriculture and Technology, Bhubaneswar, Odisha, India \\ *Corresponding author
}

\begin{tabular}{|c|c|}
\hline & A B S T R A C T \\
\hline Keywords & \multirow{4}{*}{$\begin{array}{l}\text { A poly-bag experiment was carried out during March to May } 2017 \text { in 'West } \\
\text { Central Table Land Zone', Odisha, India in complete randomized design with fly } \\
\text { ash, vermicompost and virgin soil each at } 0 \%, 20 \%, 40 \%, 60 \%, 80 \% \text { and } 100 \% \text { by } \\
\text { weight to study their effects on the floristic dynamics of the weeds in rice nursery } \\
\text { soil. Fly ash and vermicompost at different levels in rice-nursery have their } \\
\text { significant influences on the floristic composition of weeds. No broad leaf weed or } \\
\text { grass or sedge could emerge in rice nursery in absence of vermicompost except in } \\
100 \% \text { soil with only very limited weed flora. The maximum number of broadleaf } \\
\text { weeds emerged in soil with } 80 \% \text { vermicompost and the maximum number of } \\
\text { grasses and sedges emerged in } 100 \% \text { vermicompost and also in soil with } 60 \% \\
\text { vermicompost by weight. The highest dry matter of weeds was recorded in } 100 \% \\
\text { vermicompost. }\end{array}$} \\
\hline $\begin{array}{l}\text { Fly ash, } \\
\text { Vermicompost, Rice } \\
\text { nursery, Floristic } \\
\text { dynamics, Weeds, } \\
\text { Grass, Sedge, Broad } \\
\text { leaf. }\end{array}$ & \\
\hline Article Info & \\
\hline $\begin{array}{l}\text { Accepted: } \\
\text { 26 October } 2017 \\
\text { Available Online: } \\
\text { 10 December } 2017\end{array}$ & \\
\hline
\end{tabular}

\section{Introduction}

Fly ash, a necessary evil being produced to the tune of 200 MT in 2015 (Parab et al., 2012) in 180 thermal power plants in India for generation of about 192,168.88 MW energy (Central Electricity Authority, 2016) needs safe, scientific and productive use in agriculture field like many developed and developing countries of the world. Researchers are in view of its application in lower concentrations only keeping in mind the adverse microbial activities and availability of plant nutrients at higher concentration. Its significance in ameliorating the physicochemical properties of soil is widely accepted by the researchers and hence the soil fertility and crop yield increase (Rautaray et al., 2003).

Although a lot of research on the productivity and profitability of fly ash and vermicompost application at varying levels has already been done in many crops including rice but studies on the floristic dynamics of weeds were lagging behind. In this context, a poly-bag rice culture experiment was conducted to study the floristic dynamics of weeds with varying levels of fly ash and vermicompost amendments. 


\section{Materials and Methods Experimental site and climate}

The experiment was conducted during March to May 2017 in the campus of the College of Agriculture (OUAT), Chiplima, Sambalpur district, Odisha, India under Agro-climatic zone of 'West Central Table Land Zone' at around $365 \mathrm{~km}$ air distance from the Bay of Bengal at East. The experiment-site in particular was located at $83^{\circ} 53^{\prime} \mathrm{E}$ longitude, $21^{0} 21^{\prime} \mathrm{N}$ latitude and $150.75 \mathrm{~m}$ above the mean sea level experiencing tropical warm and dry climate with much rainier summers and normal to chilling winter. The long term average temperature is $26.8{ }^{\circ} \mathrm{C}$ and the average annual rainfall is $1638 \mathrm{~mm}$.

\section{Experimental design and treatments}

The treatments consisted of 3 different types of substrates i.e. virgin soil, vermicompost and fly ash. (S, V and F) at 6 different concentrations i.e. $0 \%, 20 \%, 40 \%, 60 \%, 80 \%$ and $100 \%$ leading to a total of 21 different treatment combinations (Table 2) that were replicated thrice $\left(\mathrm{R}_{1}, \mathrm{R}_{2}\right.$ and $\left.\mathrm{R}_{3}\right)$ with complete randomized design in poly-bags of $15 \mathrm{~cm}$ (diameter) and $30 \mathrm{~cm}$ height. The physicochemical properties of the above 3 treatments are as details in Table 1.

\section{Description of test-genotype used}

The test paddy variety Vijetha (MTU-1001) was developed at ANGARAU, Andhra Pradesh by crossing Krishnaveni x IR-64 and subsequently released by the OSVRC and notified in 2005. It is a semi dwarf $(115 \mathrm{~cm})$, non-lodging and medium duration (135 days) with moderate tillering habit but tolerant to flash flood and excess water situations. It has non-shattering habit with long slender, nonaromatic translucent grains with good milling, cooking and eating qualities. The yield potential of this hybrid is $5 \mathrm{tha}^{-1}$ in kharif and $5.5 \mathrm{tha}^{-1}$ in rabi.

Manures and fertilizers applied

No inorganic or organic fertilizer was added to the experimental base material except the treatments (organic soil, vermicompost and fly ash) so as to exclude their effects on results. The site from where the soil was removed and brought for experimentation had no previous cropping for last five years and thus may be delineated under "Organic zone" as per the norms of National Programme on Organic Production (NPOP). Fly ash from the nearby HINDALCO industries Ltd., Hirakud, Sambalpur was used for this purpose at different levels along with the organic soil and vermicopmost. The vermicopmost prepared by using epigeic earthworms (Eisenia fetida) in tank from farm yard manure was used for this purpose at different levels.

\section{Agronomic management practices}

The moisture content of the substrates i.e. virgin-organic soil, vermicompost and fly ash was determined by using gravimetric method. The soil, vermicompost and fly ash were mixed by weight basis according to the proportions in each treatment combinations and put inside good quality black coloured HDPE poly-bags. One hole was made in each poly-bag so as to facilitate the drainage of excess water from the substrates. The bags were kept on the ground arranged according to the replications with proper labeling. 50 numbers of good quality rice seeds of cv. MTU-1001 were selected and sterilised with $0.1 \%$ mercuric chloride for 5 minutes to avoid fungal contamination and then cleaned with distilled water for 3 times and soaked in water for 5 hours. The soaked seeds were evenly sown in the poly-bags by using a dibbler and then covered with substrates. Immediately after sowing, $300 \mathrm{~mL}$ distilled water was 
sprinkled over it.

After 10 days, seedlings were uprooted leaving only 10 healthy seedlings per polybag at uniform distance so as to avoid overcrowding at later stages. Two hand weedings at 20 days intervals i.e. 20 and 40 DAS were carried out. Distilled water of $300 \mathrm{~mL}$ was applied to the poly bags daily during the entire experimental period and adequate precautions were taken to avoid excess irrigation resulting in water stagnation. No plant protection measure was taken intentionally to study the effect of the treatments on disease-pest incidence.

\section{Methods of recording observations}

\section{Weed floristic composition}

Floristic studies of weeds (grasses, sedges and broad leaf weeds) were studied at 20, 30 and 40 DAS in detail and their common and scientific names were noted down against each treatment.

\section{Dry matter of weeds}

Dry matter of weeds was measured by air drying of the weeds collected from each polybag separately from each treatment and replication by uprooting and washing carefully. Then the weeds were oven-dried inside paper envelopes at $70{ }^{0} \mathrm{C}$ for 48 hours. The final weight was measured and expressed in $\mathrm{g} \mathrm{m}^{-2}$.

\section{Statistical analyses}

All the data obtained were statistically analyzed using F-test as per the procedure suggested by Gomez and Gomez (1984). Least significant difference (LSD) values at $\mathrm{p}=0.05$ were used to determine the significant differences between treatment means.

\section{Results and Discussion}

Studies on floristic composition and dry matter of weeds were carried out at 20,30 and 40 DAS in all treatment combinations.

\section{Number of broad leaf weeds}

The number of broad leaf weeds $\mathrm{m}^{-2}$ counted at 20,30 and 40 DAS have been presented in Table 3 and depicted in Figure 1. The population of broad leaf weeds was significantly the highest $(640.9 \pm 70.5)$ in $\mathrm{S}_{40} \mathrm{~F}_{0} \mathrm{~V}_{60}$ at 20 DAS but at subsequent two dates of observations, the population of such type of weeds in $\mathrm{S}_{20} \mathrm{~F}_{0} \mathrm{~V}_{80}$ increased and surpassed the former one with significant differences. No broad leaf weed was seen in $\mathrm{S}_{0} \mathrm{~F}_{80} \mathrm{~V}_{20}, \quad \mathrm{~S}_{0} \mathrm{~F}_{100} \mathrm{~V}_{0}, \quad \mathrm{~S}_{20} \mathrm{~F}_{80} \mathrm{~V}_{0}, \quad \mathrm{~S}_{40} \mathrm{~F}_{60} \mathrm{~V}_{0}$, $\mathrm{S}_{60} \mathrm{~F}_{40} \mathrm{~V}_{0}$ and $\mathrm{S}_{80} \mathrm{~F}_{20} \mathrm{~V}_{0}$ throughout the seedling growth. But, in $\mathrm{S}_{20} \mathrm{~F}_{60} \mathrm{~V}_{20}$ and $\mathrm{S}_{40} \mathrm{~F}_{40} \mathrm{~V}_{20}$ although no broad leaf weed was seen at 20 or 40 DAS but some of these weeds emerged at 30 DAS. Broad leaf weeds that were present in $\mathrm{S}_{0} \mathrm{~F}_{60} \mathrm{~V}_{40}$ at 20 and 30 DAS perished at 40 DAS.

\section{Number of grasses and sedges}

The number of grasses and sedges $\mathrm{m}^{-2}$ other than rice seedlings at 20,30 and 40 DAS have been presented in Table 4 and depicted in Figure 2. The population of grasses and sedges in $\mathrm{S}_{60} \mathrm{~F}_{0} \mathrm{~V}_{40}(584.3+35.1)$ was although equal to $\mathrm{S}_{40} \mathrm{~F}_{0} \mathrm{~V}_{60}(584.3+35.1)$ at 20 DAS but the population in former treatment combination reached at its peak (1036.8 \pm 82.9$)$ surpassing the latter one with significant difference at 30 DAS. However, such weed population at 40 DAS in $\mathrm{S}_{60} \mathrm{~F}_{0} \mathrm{~V}_{40}$ reduced well below $\mathrm{S}_{40} \mathrm{~F}_{0} \mathrm{~V}_{60}$ as the latter could accommodate significantly the highest number of grasses and sedges at this stage. No grass or sedge was seen in $\mathrm{S}_{0} \mathrm{~F}_{100} \mathrm{~V}_{0}, \mathrm{~S}_{20} \mathrm{~F}_{80} \mathrm{~V}_{0}$, $\mathrm{S}_{40} \mathrm{~F}_{60} \mathrm{~V}_{0}, \mathrm{~S}_{60} \mathrm{~F}_{40} \mathrm{~V}_{0}$ and $\mathrm{S}_{80} \mathrm{~F}_{20} \mathrm{~V}_{0}$ at all 3 dates of observations except in the last one where $75.2+6.8$ numbers of such weeds were seen 
at 40 DAS.

\section{Total number of weeds}

The total number weeds $\mathrm{m}^{-2}$ counted at 20, 30 and 40 DAS as presented in Table 5 and depicted in Figure 3 revealed that $\mathrm{S}_{40} \mathrm{~F}_{0} \mathrm{~V}_{60}$ could accommodate significantly the highest number of weeds $(1225.2 \pm 123.1)$ followed by $\mathrm{S}_{60} \mathrm{~F}_{0} \mathrm{~V}_{40}(904.5 \pm 82.0)$ at 20 DAS but after 10 days elapse the latter surpassed the former one by accommodating significantly the highest numbers of weeds (1677.7 \pm 134.2). Although the total weed population in $\mathrm{S}_{40} \mathrm{~F}_{0} \mathrm{~V}_{60}$ decreased with the progress in rice seedling age from 20 to 40 days but at 40 DAS it had the highest population followed by $\mathrm{S}_{20} \mathrm{~F}_{0} \mathrm{~V}_{80}, \mathrm{~S}_{40} \mathrm{~F}_{20} \mathrm{~V}_{40}, \mathrm{~S}_{0} \mathrm{~F}_{0} \mathrm{~V}_{100}$ and $\mathrm{S}_{60} \mathrm{~F}_{0} \mathrm{~V}_{40}$ without any statistical difference between the two former and three latter combinations.

No broadleaf or grass or sedge was seen in $\mathrm{S}_{0} \mathrm{~F}_{100} \mathrm{~V}_{0}, \quad \mathrm{~S}_{20} \mathrm{~F}_{80} \mathrm{~V}_{0}, \quad \mathrm{~S}_{40} \mathrm{~F}_{60} \mathrm{~V}_{0}, \quad \mathrm{~S}_{60} \mathrm{~F}_{40} \mathrm{~V}_{0}$ and $\mathrm{S}_{80} \mathrm{~F}_{20} \mathrm{~V}_{0}$ at all three dates of observations except in $\mathrm{S}_{80} \mathrm{~F}_{20} \mathrm{~V}_{0}$ having some grasses and sedges only at 40 DAS.

Positive effect of vermicompost on weed population could be due to availability of favourable growing medium so also the weed propagules in it. But, in fly ash, due to unfavourable soil physicochemical properties and absence of such propagules, the weed population was either very marginal or absent.

Table.1 Physico-chemical properties of treatments

\begin{tabular}{|c|c|c|c|c|c|c|c|c|c|}
\hline $\begin{array}{l}\text { Type of } \\
\text { substrates }\end{array}$ & $\mathbf{p H}$ & \multicolumn{2}{|c|}{ Sand (\%) } & \multicolumn{2}{|c|}{ Silt (\%) } & \multicolumn{2}{|c|}{ Clay $(\%)$} & $\begin{array}{c}\text { Organic } \\
\text { carbon (\%) }\end{array}$ & $\begin{array}{c}\mathrm{EC} \\
\left(\mathrm{dS} \mathrm{m^{-1 }}\right)\end{array}$ \\
\hline Vermicompost & 6.63 & \multicolumn{2}{|c|}{42.4} & \multicolumn{2}{|c|}{8.0} & & 49.6 & 0.360 & 0.62 \\
\hline Fly ash & 6.43 & \multicolumn{2}{|c|}{10.4} & \multicolumn{2}{|c|}{36.0} & & 53.6 & 0.090 & 0.11 \\
\hline Virgin soil & 6.71 & \multicolumn{2}{|c|}{22.4} & \multicolumn{2}{|c|}{8.0} & & 69.6 & 0.018 & 0.18 \\
\hline \multirow{2}{*}{$\begin{array}{l}\text { Type of } \\
\text { substrates }\end{array}$} & \multicolumn{9}{|c|}{ Available } \\
\hline & \multicolumn{2}{|c|}{$\begin{array}{c}\mathrm{N} \\
\left(\mathrm{kg} \mathrm{ha}^{-1}\right)\end{array}$} & \multicolumn{2}{|c|}{$\begin{array}{c}\mathrm{P}_{2} \mathrm{O}_{5} \\
\left(\mathrm{~kg} \mathrm{ha}^{-1}\right)\end{array}$} & \multicolumn{2}{|c|}{$\begin{array}{c}\mathrm{K}_{2} \mathrm{O} \\
\left(\mathrm{kg} \mathrm{ha}^{-1}\right)\end{array}$} & $\begin{array}{c}\mathrm{S} \\
\left(\mathrm{mg} \mathrm{kg}^{-1}\right)\end{array}$ & $\begin{array}{c}\text { B } \\
\left(\mathrm{mg} \mathrm{kg}^{-1}\right)\end{array}$ & $\begin{array}{c}\mathrm{Zn} \\
\left(\mathrm{mg} \mathrm{kg}^{-1}\right)\end{array}$ \\
\hline Vermicompost & \multicolumn{2}{|c|}{231.2} & \multicolumn{2}{|c|}{280.3} & \multicolumn{2}{|c|}{598.4} & 204.730 & 9.657 & 2.92 \\
\hline Fly ash & \multicolumn{2}{|c|}{115.3} & \multicolumn{2}{|c|}{277.4} & \multicolumn{2}{|c|}{348.9} & 89.526 & 0.577 & 2.98 \\
\hline Virgin soil & \multicolumn{2}{|c|}{110.4} & \multicolumn{2}{|c|}{72.6} & \multicolumn{2}{|c|}{358.7} & 0.347 & 0.022 & 2.82 \\
\hline
\end{tabular}

Table.2 Details of treatment combinations and symbols used 


\section{Sl. No.}

1 Soil $(0 \%)+$ Fly ash $(0 \%)+$ Vermicompost $(100 \%)$

$\mathrm{S}_{0} \mathrm{~F}_{0} \mathrm{~V}_{100}$

2 Soil $(0 \%)+$ Fly ash $(20 \%)+$ Vermicompost (80\%)

$\mathrm{S}_{0} \mathrm{~F}_{20} \mathrm{~V}_{80}$

3 Soil $(0 \%)+$ Fly ash $(40 \%)+$ Vermicompost (60\%)

$\mathrm{S}_{0} \mathrm{~F}_{40} \mathrm{~V}_{60}$

4 Soil $(0 \%)+$ Fly ash $(60 \%)+$ Vermicompost (40\%)

$\mathrm{S}_{0} \mathrm{~F}_{60} \mathrm{~V}_{40}$

5 Soil $(0 \%)+$ Fly ash $(80 \%)+$ Vermicompost (20\%)

$\mathrm{S}_{0} \mathrm{~F}_{80} \mathrm{~V}_{20}$

6 Soil $(0 \%)+$ Fly ash $(100 \%)+$ Vermicompost $(0 \%)$

$\mathrm{S}_{0} \mathrm{~F}_{100} \mathrm{~V}_{0}$

7 Soil $(20 \%)+$ Fly ash $(0 \%)+$ Vermicompost (80\%)

$\mathrm{S}_{20} \mathrm{~F}_{0} \mathrm{~V}_{80}$

8 Soil $(20 \%)+$ Fly ash $(20 \%)+$ Vermicompost $(60 \%)$

$\mathrm{S}_{20} \mathrm{~F}_{20} \mathrm{~V}_{60}$

9 Soil $(20 \%)+$ Fly ash $(40 \%)+$ Vermicompost $(40 \%)$

$\mathrm{S}_{20} \mathrm{~F}_{40} \mathrm{~V}_{40}$

10 Soil $(20 \%)+$ Fly ash $(60 \%)+$ Vermicompost $(20 \%)$

$\mathrm{S}_{20} \mathrm{~F}_{60} \mathrm{~V}_{20}$

11 Soil $(20 \%)+$ Fly ash $(80 \%)+$ Vermicompost (0\%)

$\mathrm{S}_{20} \mathrm{~F}_{80} \mathrm{~V}_{0}$

12 Soil $(40 \%)+$ Fly ash $(0 \%)+$ Vermicompost (60\%)

$\mathrm{S}_{40} \mathrm{~F}_{0} \mathrm{~V}_{60}$

13 Soil $(40 \%)+$ Fly ash $(20 \%)+$ Vermicompost $(40 \%)$

$\mathrm{S}_{40} \mathrm{~F}_{20} \mathrm{~V}_{40}$

14 Soil $(40 \%)+$ Fly ash $(40 \%)+$ Vermicompost (20\%)

$\mathrm{S}_{40} \mathrm{~F}_{40} \mathrm{~V}_{20}$

15 Soil $(40 \%)+$ Fly ash $(60 \%)+$ Vermicompost $(0 \%)$

$\mathrm{S}_{40} \mathrm{~F}_{60} \mathrm{~V}_{0}$

16 Soil $(60 \%)+$ Fly ash $(0 \%)+$ Vermicompost (40\%)

$\mathrm{S}_{60} \mathrm{~F}_{0} \mathrm{~V}_{40}$

17 Soil $(60 \%)+$ Fly ash (20\%) + Vermicompost (20\%)

$\mathrm{S}_{60} \mathrm{~F}_{20} \mathrm{~V}_{20}$

18 Soil $(60 \%)+$ Fly ash $(40 \%)+$ Vermicompost $(0 \%)$

$\mathrm{S}_{60} \mathrm{~F}_{40} \mathrm{~V}_{0}$

19 Soil $(80 \%)+$ Fly ash $(0 \%)+$ Vermicompost (20\%)

$\mathrm{S}_{80} \mathrm{~F}_{0} \mathrm{~V}_{20}$

20 Soil $(80 \%)+$ Fly ash $(20 \%)+$ Vermicompost $(0 \%)$

$\mathrm{S}_{80} \mathrm{~F}_{20} \mathrm{~V}_{0}$

21 Soil $(100 \%)+$ Fly ash $(0 \%)+$ Vermicompost (0\%)

$\mathrm{S}_{100} \mathrm{~F}_{0} \mathrm{~V}_{0}$

Table.3 Effect of treatment combinations on number of broad leaf weeds $\mathrm{m}^{-2}$ at different growth stages of rice seedlings 


\begin{tabular}{|c|c|c|c|c|}
\hline \multirow{2}{*}{$\begin{array}{l}\text { Treatment } \\
\text { combinations }\end{array}$} & \multirow{2}{*}{$\begin{array}{l}\text { Sl. } \\
\text { No. }\end{array}$} & \multicolumn{3}{|c|}{ Days after sowing } \\
\hline & & 20 & 30 & 40 \\
\hline $\mathrm{S}_{0} \mathrm{~F}_{0} \mathrm{~V}_{100}$ & 1 & $320.2 \pm 25.6$ & $452.5 \pm 49.8$ & $131.8 \pm 10.5$ \\
\hline $\mathrm{S}_{0} \mathrm{~F}_{20} \mathrm{~V}_{80}$ & 2 & $93.9 \pm 4.7$ & $93.9 \pm 11.3$ & $75.2 \pm 3.8$ \\
\hline $\mathrm{S}_{0} \mathrm{~F}_{40} \mathrm{~V}_{60}$ & 3 & $150.5 \pm 16.6$ & $75.2 \pm 8.3$ & $17.0 \pm 1.9$ \\
\hline $\mathrm{S}_{0} \mathrm{~F}_{60} \mathrm{~V}_{40}$ & 4 & $39.6 \pm 3.6$ & $75.2 \pm 10.5$ & $0.0 \pm 0.0$ \\
\hline $\mathrm{S}_{0} \mathrm{~F}_{80} \mathrm{~V}_{20}$ & 5 & $0.0 \pm 0.0$ & $0.0 \pm 0.0$ & $0.0 \pm 0.0$ \\
\hline $\mathrm{S}_{0} \mathrm{~F}_{100} \mathrm{~V}_{0}$ & 6 & $0.0 \pm 0.0$ & $0.0 \pm 0.0$ & $0.0 \pm 0.0$ \\
\hline $\mathrm{S}_{20} \mathrm{~F}_{0} \mathrm{~V}_{80}$ & 7 & $433.3 \pm 39.0$ & $810.6 \pm 73.0$ & $469.5 \pm 42.3$ \\
\hline $\mathrm{S}_{20} \mathrm{~F}_{20} \mathrm{~V}_{60}$ & 8 & $93.9 \pm 8.5$ & $188.4 \pm 17.0$ & $243.2 \pm 21.9$ \\
\hline $\mathrm{S}_{20} \mathrm{~F}_{40} \mathrm{~V}_{40}$ & 9 & $131.8 \pm 13.2$ & $188.4 \pm 9.4$ & $93.9 \pm 9.4$ \\
\hline $\mathrm{S}_{20} \mathrm{~F}_{60} \mathrm{~V}_{20}$ & 10 & $0.0 \pm 0.0$ & $56.6 \pm 6.8$ & $75.2 \pm 9.0$ \\
\hline $\mathrm{S}_{20} \mathrm{~F}_{80} \mathrm{~V}_{0}$ & 11 & $0.0 \pm 0.0$ & $0.0 \pm 0.0$ & $0.0 \pm 0.0$ \\
\hline $\mathrm{S}_{40} \mathrm{~F}_{0} \mathrm{~V}_{60}$ & 12 & $640.9 \pm 70.5$ & $640.9 \pm 57.7$ & $358.1 \pm 39.4$ \\
\hline $\mathrm{S}_{40} \mathrm{~F}_{20} \mathrm{~V}_{40}$ & 13 & $37.3 \pm 3.4$ & $188.4 \pm 15.1$ & $244.9 \pm 22.0$ \\
\hline $\mathrm{S}_{40} \mathrm{~F}_{40} \mathrm{~V}_{20}$ & 14 & $0.0 \pm 0.0$ & $18.7 \pm 1.1$ & $0.0 \pm 0.0$ \\
\hline $\mathrm{S}_{40} \mathrm{~F}_{60} \mathrm{~V}_{0}$ & 15 & $0.0 \pm 0.0$ & $0.0 \pm 0.0$ & $0.0 \pm 0.0$ \\
\hline $\mathrm{S}_{60} \mathrm{~F}_{0} \mathrm{~V}_{40}$ & 16 & $320.2 \pm 35.2$ & $640.9 \pm 51.3$ & $301.5 \pm 33.2$ \\
\hline $\mathrm{S}_{60} \mathrm{~F}_{20} \mathrm{~V}_{20}$ & 17 & $188.4 \pm 18.8$ & $207.0 \pm 14.5$ & $301.5 \pm 30.1$ \\
\hline $\mathrm{S}_{60} \mathrm{~F}_{40} \mathrm{~V}_{0}$ & 18 & $0.0 \pm 0.0$ & $0.0 \pm 0.0$ & $0.0 \pm 0.0$ \\
\hline $\mathrm{S}_{80} \mathrm{~F}_{0} \mathrm{~V}_{20}$ & 19 & $188.4 \pm 17.0$ & $263.6 \pm 23.7$ & $301.5 \pm 27.1$ \\
\hline $\mathrm{S}_{80} \mathrm{~F}_{20} \mathrm{~V}_{0}$ & 20 & $0.0 \pm 0.0$ & $0.0 \pm 0.0$ & $0.0 \pm 0.0$ \\
\hline $\mathrm{S}_{100} \mathrm{~F}_{0} \mathrm{~V}_{0}$ & 21 & $131.8 \pm 15.8$ & $100.1 \pm 7.0$ & $93.9 \pm 11.3$ \\
\hline S.Em (+) & & 12.5 & 15.7 & 10.9 \\
\hline C.D. (0.05) & & 35.7 & 44.8 & 31.1 \\
\hline C.V. $(\%)$ & & 128.5 & 125.7 & 113.6 \\
\hline
\end{tabular}


Table.4 Effect of treatment combinations on number of grasses and sedges $\mathrm{m}^{-2}$ at different growth stages of rice seedlings

\begin{tabular}{|c|c|c|c|c|}
\hline \multirow{2}{*}{$\begin{array}{l}\text { Treatment } \\
\text { combinations }\end{array}$} & \multirow{2}{*}{ Sl. No. } & \multicolumn{3}{|c|}{ Days after sowing } \\
\hline & & 20 & 30 & 40 \\
\hline $\mathrm{S}_{0} \mathrm{~F}_{0} \mathrm{~V}_{100}$ & 1 & $301.5 \pm 12.1$ & $735.4 \pm 80.9$ & $527.8 \pm 42.2$ \\
\hline $\mathrm{S}_{0} \mathrm{~F}_{20} \mathrm{~V}_{80}$ & 2 & $433.3 \pm 26.0$ & $339.4 \pm 40.7$ & $320.2 \pm 16.0$ \\
\hline $\mathrm{S}_{0} \mathrm{~F}_{40} \mathrm{~V}_{60}$ & 3 & $301.5 \pm 18.1$ & $244.9 \pm 26.9$ & $243.2 \pm 26.8$ \\
\hline $\mathrm{S}_{0} \mathrm{~F}_{60} \mathrm{~V}_{40}$ & 4 & $150.5 \pm 7.5$ & $207.0 \pm 29.0$ & $188.4 \pm 17.0$ \\
\hline $\mathrm{S}_{0} \mathrm{~F}_{80} \mathrm{~V}_{20}$ & 5 & $37.3 \pm 1.9$ & $75.2 \pm 5.3$ & $150.5 \pm 10.5$ \\
\hline $\mathrm{S}_{0} \mathrm{~F}_{100} \mathrm{~V}_{0}$ & 6 & $0.0 \pm 0.0$ & $0.0 \pm 0.0$ & $0.0 \pm 0.0$ \\
\hline $\mathrm{S}_{20} \mathrm{~F}_{0} \mathrm{~V}_{80}$ & 7 & $433.3 \pm 26.0$ & $376.7 \pm 33.9$ & $358.1 \pm 32.2$ \\
\hline $\mathrm{S}_{20} \mathrm{~F}_{20} \mathrm{~V}_{60}$ & 8 & $301.5 \pm 18.1$ & $301.5 \pm 27.1$ & $320.2 \pm 28.8$ \\
\hline $\mathrm{S}_{20} \mathrm{~F}_{40} \mathrm{~V}_{40}$ & 9 & $75.2 \pm 4.5$ & $75.2 \pm 3.8$ & $188.4 \pm 18.8$ \\
\hline $\mathrm{S}_{20} \mathrm{~F}_{60} \mathrm{~V}_{20}$ & 10 & $131.8 \pm 9.2$ & $93.9 \pm 11.3$ & $75.2 \pm 9.0$ \\
\hline $\mathrm{S}_{20} \mathrm{~F}_{80} \mathrm{~V}_{0}$ & 11 & $0.0 \pm 0.0$ & $0.0 \pm 0.0$ & $0.0 \pm 0.0$ \\
\hline $\mathrm{S}_{40} \mathrm{~F}_{0} \mathrm{~V}_{60}$ & 12 & $584.3 \pm 35.1$ & $527.8 \pm 47.5$ & $527.8 \pm 58.1$ \\
\hline $\mathrm{S}_{40} \mathrm{~F}_{20} \mathrm{~V}_{40}$ & 13 & $489.9 \pm 29.4$ & $433.3 \pm 34.7$ & $433.3 \pm 39.0$ \\
\hline $\mathrm{S}_{40} \mathrm{~F}_{40} \mathrm{~V}_{20}$ & 14 & $93.9 \pm 3.8$ & $150.5 \pm 9.0$ & $207.0 \pm 24.8$ \\
\hline $\mathrm{S}_{40} \mathrm{~F}_{60} \mathrm{~V}_{0}$ & 15 & $0.0 \pm 0.0$ & $0.0 \pm 0.0$ & $0.0 \pm 0.0$ \\
\hline $\mathrm{S}_{60} \mathrm{~F}_{0} \mathrm{~V}_{40}$ & 16 & $584.3 \pm 35.1$ & $1036.8 \pm 82.9$ & $358.1 \pm 39.4$ \\
\hline $\mathrm{S}_{60} \mathrm{~F}_{20} \mathrm{~V}_{20}$ & 17 & $188.4 \pm 11.3$ & $244.9 \pm 17.1$ & $301.5 \pm 30.1$ \\
\hline $\mathrm{S}_{60} \mathrm{~F}_{40} \mathrm{~V}_{0}$ & 18 & $0.0 \pm 0.0$ & $0.0 \pm 0.0$ & $0.0 \pm 0.0$ \\
\hline $\mathrm{S}_{80} \mathrm{~F}_{0} \mathrm{~V}_{20}$ & 19 & $263.6 \pm 13.2$ & $414.6 \pm 37.3$ & $358.1 \pm 32.2$ \\
\hline $\mathrm{S}_{80} \mathrm{~F}_{20} \mathrm{~V}_{0}$ & 20 & $0.0 \pm 0.0$ & $0.0 \pm 0.0$ & $75.2 \pm 6.8$ \\
\hline $\mathrm{S}_{100} \mathrm{~F}_{0} \mathrm{~V}_{0}$ & 21 & $37.3 \pm 1.9$ & $93.9 \pm 6.6$ & $75.2 \pm 9.0$ \\
\hline S.Em (+) & & 9.7 & 19.5 & 15.2 \\
\hline C.D. (0.05) & & 27.8 & 55.7 & 43.4 \\
\hline C.V. (\%) & & 94.4 & 104.6 & 75.5 \\
\hline
\end{tabular}


Table.5 Effect of treatment combinations on total number of weeds $\mathrm{m}^{-2}$ at different growth stages of rice seedlings

\begin{tabular}{|c|c|c|c|c|}
\hline \multirow{2}{*}{$\begin{array}{l}\text { Treatment } \\
\text { combinations }\end{array}$} & \multirow{2}{*}{$\begin{array}{l}\text { Sl. } \\
\text { No. }\end{array}$} & \multicolumn{3}{|c|}{ Days after sowing } \\
\hline & & 20 & 30 & 40 \\
\hline $\mathrm{S}_{0} \mathrm{~F}_{0} \mathrm{~V}_{100}$ & 1 & $621.7 \pm 68.4$ & $1187.9 \pm 130.7$ & $659.6 \pm 52.8$ \\
\hline $\mathrm{S}_{0} \mathrm{~F}_{20} \mathrm{~V}_{80}$ & 2 & $527.2 \pm 56.7$ & $433.3 \pm 52.0$ & $395.4 \pm 19.8$ \\
\hline $\mathrm{S}_{0} \mathrm{~F}_{40} \mathrm{~V}_{60}$ & 3 & $452.0 \pm 49.7$ & $320.2 \pm 35.2$ & $260.2 \pm 28.6$ \\
\hline $\mathrm{S}_{0} \mathrm{~F}_{60} \mathrm{~V}_{40}$ & 4 & $150.5 \pm 21.1$ & $282.3 \pm 39.5$ & $188.4 \pm 17.0$ \\
\hline $\mathrm{S}_{0} \mathrm{~F}_{80} \mathrm{~V}_{20}$ & 5 & $74.7 \pm 5.2$ & $75.2 \pm 5.3$ & $150.5 \pm 10.5$ \\
\hline $\mathrm{S}_{0} \mathrm{~F}_{100} \mathrm{~V}_{0}$ & 6 & $0.0 \pm 0.0$ & $0.0 \pm 0.0$ & $0.0 \pm 0.0$ \\
\hline $\mathrm{S}_{20} \mathrm{~F}_{0} \mathrm{~V}_{80}$ & 7 & $866.6 \pm 78.0$ & $1187.3 \pm 106.9$ & $827.6 \pm 74.5$ \\
\hline $\mathrm{S}_{20} \mathrm{~F}_{20} \mathrm{~V}_{60}$ & 8 & $395.4 \pm 35.6$ & $489.9 \pm 44.1$ & $563.4 \pm 50.7$ \\
\hline $\mathrm{S}_{20} \mathrm{~F}_{40} \mathrm{~V}_{40}$ & 9 & $207.0 \pm 20.9$ & $263.6 \pm 13.2$ & $282.3 \pm 28.2$ \\
\hline $\mathrm{S}_{20} \mathrm{~F}_{60} \mathrm{~V}_{20}$ & 10 & $131.8 \pm 15.8$ & $150.5 \pm 18.1$ & $150.5 \pm 18.1$ \\
\hline $\mathrm{S}_{20} \mathrm{~F}_{80} \mathrm{~V}_{0}$ & 11 & $0.0 \pm 0.0$ & $0.0 \pm 0.0$ & $0.0 \pm 0.0$ \\
\hline $\mathrm{S}_{40} \mathrm{~F}_{0} \mathrm{~V}_{60}$ & 12 & $1225.2 \pm 123.1$ & $1168.6 \pm 105.2$ & $885.8 \pm 97.4$ \\
\hline $\mathrm{S}_{40} \mathrm{~F}_{20} \mathrm{~V}_{40}$ & 13 & $527.2 \pm 42.5$ & $621.7 \pm 49.7$ & $678.2 \pm 61.0$ \\
\hline $\mathrm{S}_{40} \mathrm{~F}_{40} \mathrm{~V}_{20}$ & 14 & $93.9 \pm 5.6$ & $169.1 \pm 10.1$ & $207.0 \pm 24.8$ \\
\hline $\mathrm{S}_{40} \mathrm{~F}_{60} \mathrm{~V}_{0}$ & 15 & $0.0 \pm 0.0$ & $0.0 \pm 0.0$ & $0.0 \pm 0.0$ \\
\hline $\mathrm{S}_{60} \mathrm{~F}_{0} \mathrm{~V}_{40}$ & 16 & $904.5 \pm 82.0$ & $1677.7 \pm 134.2$ & $659.6 \pm 72.6$ \\
\hline $\mathrm{S}_{60} \mathrm{~F}_{20} \mathrm{~V}_{20}$ & 17 & $376.7 \pm 32.0$ & $452.0 \pm 31.6$ & $603.0 \pm 60.3$ \\
\hline $\mathrm{S}_{60} \mathrm{~F}_{40} \mathrm{~V}_{0}$ & 18 & $0.0 \pm 0.0$ & $0.0 \pm 0.0$ & $0.0 \pm 0.0$ \\
\hline $\mathrm{S}_{80} \mathrm{~F}_{0} \mathrm{~V}_{20}$ & 19 & $452.0 \pm 40.7$ & $678.2 \pm 61.0$ & $659.6 \pm 59.4$ \\
\hline $\mathrm{S}_{80} \mathrm{~F}_{20} \mathrm{~V}_{0}$ & 20 & $0.0 \pm 0.0$ & $0.0 \pm 0.0$ & $75.2 \pm 6.8$ \\
\hline $\mathrm{S}_{100} \mathrm{~F}_{0} \mathrm{~V}_{0}$ & 21 & $169.1 \pm 18.4$ & $194.0 \pm 13.6$ & $169.1 \pm 20.3$ \\
\hline S.Em (+) & & 26.9 & 34.0 & 25.3 \\
\hline C.D. (0.05) & & 76.8 & 97.2 & 72.2 \\
\hline C.V. $(\%)$ & & 100.2 & 107.2 & 83.2 \\
\hline
\end{tabular}


Table.6 Effect of treatment combinations on dry matter $\left(\mathrm{g} \mathrm{m}^{-2}\right)$ of weeds $\mathrm{m}^{-2}$ at different growth stages of rice seedlings

\begin{tabular}{|c|c|c|c|c|}
\hline \multirow{2}{*}{$\begin{array}{l}\text { Treatment } \\
\text { combinations }\end{array}$} & \multirow{2}{*}{ Sl. No. } & \multicolumn{3}{|c|}{ Days after sowing } \\
\hline & & 20 & 30 & 40 \\
\hline $\mathrm{S}_{0} \mathrm{~F}_{0} \mathrm{~V}_{100}$ & 1 & $2.885 \pm 0.231$ & $22.004 \pm 1.540$ & $312.808 \pm 34.409$ \\
\hline $\mathrm{S}_{0} \mathrm{~F}_{20} \mathrm{~V}_{80}$ & 2 & $2.772 \pm 0.139$ & $5.939 \pm 0.535$ & $36.598 \pm 4.392$ \\
\hline $\mathrm{S}_{0} \mathrm{~F}_{40} \mathrm{~V}_{60}$ & 3 & $2.602 \pm 0.286$ & $5.713 \pm 0.343$ & $2.715 \pm 0.299$ \\
\hline $\mathrm{S}_{0} \mathrm{~F}_{60} \mathrm{~V}_{40}$ & 4 & $0.848 \pm 0.076$ & $2.941 \pm 0.294$ & $23.135 \pm 3.239$ \\
\hline $\mathrm{S}_{0} \mathrm{~F}_{80} \mathrm{~V}_{20}$ & 5 & $0.453 \pm 0.032$ & $0.792 \pm 0.055$ & $1.640 \pm 0.115$ \\
\hline $\mathrm{S}_{0} \mathrm{~F}_{100} \mathrm{~V}_{0}$ & 6 & $0.000 \pm 0.000$ & $0.000 \pm 0.000$ & $0.000 \pm 0.000$ \\
\hline $\mathrm{S}_{20} \mathrm{~F}_{0} \mathrm{~V}_{80}$ & 7 & $4.469 \pm 0.402$ & $21.212 \pm 1.909$ & $138.190 \pm 12.437$ \\
\hline $\mathrm{S}_{20} \mathrm{~F}_{20} \mathrm{~V}_{60}$ & 8 & $6.788 \pm 0.611$ & $9.107 \pm 0.820$ & $85.301 \pm 7.677$ \\
\hline $\mathrm{S}_{20} \mathrm{~F}_{40} \mathrm{~V}_{40}$ & 9 & $0.566 \pm 0.057$ & $1.188 \pm 0.071$ & $3.224 \pm 0.161$ \\
\hline $\mathrm{S}_{20} \mathrm{~F}_{60} \mathrm{~V}_{20}$ & 10 & $0.283 \pm 0.034$ & $0.679 \pm 0.034$ & $0.566 \pm 0.068$ \\
\hline $\mathrm{S}_{20} \mathrm{~F}_{80} \mathrm{~V}_{0}$ & 11 & $0.000 \pm 0.000$ & $0.000 \pm 0.000$ & $0.000 \pm 0.000$ \\
\hline $\mathrm{S}_{40} \mathrm{~F}_{0} \mathrm{~V}_{60}$ & 12 & $9.333 \pm 1.027$ & $12.222 \pm 0.495$ & $126.481 \pm 11.383$ \\
\hline $\mathrm{S}_{40} \mathrm{~F}_{20} \mathrm{~V}_{40}$ & 13 & $4.242 \pm 0.382$ & $5.034 \pm 0.453$ & $35.580 \pm 2.846$ \\
\hline $\mathrm{S}_{40} \mathrm{~F}_{40} \mathrm{~V}_{20}$ & 14 & $0.792 \pm 0.095$ & $0.622 \pm 0.019$ & $3.677 \pm 0.221$ \\
\hline $\mathrm{S}_{40} \mathrm{~F}_{60} \mathrm{~V}_{0}$ & 15 & $0.000 \pm 0.000$ & $0.000 \pm 0.000$ & $0.000 \pm 0.000$ \\
\hline $\mathrm{S}_{60} \mathrm{~F}_{0} \mathrm{~V}_{40}$ & 16 & $1.640 \pm 0.180$ & $21.699 \pm 2.387$ & $33.713 \pm 2.697$ \\
\hline $\mathrm{S}_{60} \mathrm{~F}_{20} \mathrm{~V}_{20}$ & 17 & $0.283 \pm 0.028$ & $0.905 \pm 0.091$ & $31.960 \pm 2.237$ \\
\hline $\mathrm{S}_{60} \mathrm{~F}_{40} \mathrm{~V}_{0}$ & 18 & $0.000 \pm 0.000$ & $0.000 \pm 0.000$ & $0.000 \pm 0.000$ \\
\hline $\mathrm{S}_{80} \mathrm{~F}_{0} \mathrm{~V}_{20}$ & 19 & $1.810 \pm 0.163$ & $7.806 \pm 0.156$ & $101.196 \pm 9.108$ \\
\hline $\mathrm{S}_{80} \mathrm{~F}_{20} \mathrm{~V}_{0}$ & 20 & $0.000 \pm 0.000$ & $0.000 \pm 0.000$ & $6.166 \pm 0.555$ \\
\hline $\mathrm{S}_{100} \mathrm{~F}_{0} \mathrm{~V}_{0}$ & 21 & $0.735 \pm 0.088$ & $2.206 \pm 0.066$ & $7.071 \pm 0.495$ \\
\hline S.Em (+) & & 0.177 & 0.461 & 5.135 \\
\hline C.D. (0.05) & & 0.506 & 1.300 & 14.654 \\
\hline C.V. $(\%)$ & & 127.863 & 129.390 & 164.181 \\
\hline
\end{tabular}


Table.7 Floristic composition of weed species as influenced by different treatment combinations at 40 DAS of rice seedlings

\begin{tabular}{|c|c|c|c|c|}
\hline $\begin{array}{l}\text { Treatment } \\
\text { combinations }\end{array}$ & $\begin{array}{l}\text { Sl. } \\
\text { No. }\end{array}$ & Grasses & Sedges & Broadleaf \\
\hline $\mathrm{S}_{0} \mathrm{~F}_{0} \mathrm{~V}_{100}$ & 1 & $\begin{array}{l}\text { Brachiaria reptans, } \\
\text { Echinochloa colona, } \\
\text { Echinochloa crusgali, }\end{array}$ & $\begin{array}{l}\text { Cyperus difformis, } \\
\text { Cyperus rotundus }\end{array}$ & $\begin{array}{l}\text { Phyllanthus urinaria, Ludwigia } \\
\text { parviflora, Ammannia baccifera, } \\
\text { Scoparia dolcis, }\end{array}$ \\
\hline $\mathrm{S}_{0} \mathrm{~F}_{20} \mathrm{~V}_{80}$ & 2 & $\begin{array}{l}\text { Brachiaria reptans, } \\
\text { Echinochloa colona, } \\
\text { Echinochloa crusgali, }\end{array}$ & $\begin{array}{l}\text { Cyperus difformis, } \\
\text { Cyperus rotundus }\end{array}$ & $\begin{array}{l}\text { Phyllanthus niruri, Phyllanthus } \\
\text { urinaria, Ludwigia parviflora,, } \\
\text { Scoparia dolcis, }\end{array}$ \\
\hline $\mathrm{S}_{0} \mathrm{~F}_{40} \mathrm{~V}_{60}$ & 3 & $\begin{array}{l}\text { Brachiaria reptans, } \\
\text { Echinochloa colona, } \\
\text { Echinochloa crusgali, }\end{array}$ & $\begin{array}{l}\text { Cyperus difformis, } \\
\text { Cyperus rotundus }\end{array}$ & $\begin{array}{l}\text { Phyllanthus urinaria, Ludwigia } \\
\text { parviflora,, Scoparia dolcis, }\end{array}$ \\
\hline $\mathrm{S}_{0} \mathrm{~F}_{60} \mathrm{~V}_{40}$ & 4 & Echinochloa crusgali, & Cyperus difformis & - \\
\hline $\mathrm{S}_{0} \mathrm{~F}_{80} \mathrm{~V}_{20}$ & 5 & Echinochloa crusgali, & Cyperus difformis & - \\
\hline $\mathrm{S}_{0} \mathrm{~F}_{100} \mathrm{~V}_{0}$ & 6 & - & - & - \\
\hline $\mathrm{S}_{20} \mathrm{~F}_{0} \mathrm{~V}_{80}$ & 7 & $\begin{array}{c}\text { Cynodon dactylon, Brachiaria } \\
\text { reptans, Echinochloa colona, } \\
\text { Echinochloa crusgali, }\end{array}$ & $\begin{array}{l}\text { Cyperus difformis, } \\
\text { Cyperus rotundus }\end{array}$ & $\begin{array}{l}\text { Phyllanthus niruri, Phyllanthus } \\
\text { urinaria, Ludwigia parviflora, } \\
\text { Ammannia baccifera, Scoparia } \\
\text { dolcis, Chenopodium album }\end{array}$ \\
\hline $\mathrm{S}_{20} \mathrm{~F}_{20} \mathrm{~V}_{60}$ & 8 & $\begin{array}{l}\text { Cynodon dactylon, Brachiaria } \\
\text { reptans, Echinochloa colona, } \\
\text { Echinochloa crusgali, }\end{array}$ & $\begin{array}{l}\text { Cyperus difformis, } \\
\text { Cyperus rotundus }\end{array}$ & $\begin{array}{l}\text { Ludwigia parviflora, Scoparia } \\
\text { dolcis, Chenopodium album }\end{array}$ \\
\hline $\mathrm{S}_{20} \mathrm{~F}_{40} \mathrm{~V}_{40}$ & 9 & $\begin{array}{l}\text { Cynodon dactylon, Brachiaria } \\
\text { reptans, Echinochloa colona, } \\
\text { Echinochloa crusgali, }\end{array}$ & Cyperus difformis & $\begin{array}{l}\text { Phyllanthus niruri, Ludwigia } \\
\text { parviflora, Scoparia dolcis, }\end{array}$ \\
\hline $\mathrm{S}_{20} \mathrm{~F}_{60} \mathrm{~V}_{20}$ & 10 & $\begin{array}{l}\text { Brachiaria reptans, } \\
\text { Echinochloa colona, } \\
\text { Echinochloa crusgali }\end{array}$ & Cyperus difformis & $\begin{array}{l}\text { Phyllanthus niruri, Phyllanthus } \\
\text { urinaria, Scoparia dolcis }\end{array}$ \\
\hline $\mathrm{S}_{20} \mathrm{~F}_{80} \mathrm{~V}_{0}$ & 11 & - & . & - \\
\hline $\mathrm{S}_{40} \mathrm{~F}_{0} \mathrm{~V}_{60}$ & 12 & $\begin{array}{l}\text { Cynodon dactylon, Brachiaria } \\
\text { reptans, Echinochloa colona, } \\
\text { Echinochloa crusgali, }\end{array}$ & $\begin{array}{l}\text { Cyperus difformis, } \\
\text { Cyperus rotundus }\end{array}$ & $\begin{array}{l}\text { Phyllanthus niruri, Phyllanthus } \\
\text { urinaria, Ludwigia parviflora, } \\
\text { Ammannia baccifera, Scoparia } \\
\text { dolcis, Chenopodium album }\end{array}$ \\
\hline $\mathrm{S}_{40} \mathrm{~F}_{20} \mathrm{~V}_{40}$ & 13 & $\begin{array}{l}\text { Brachiaria reptans. } \\
\text { Echinochloa colona }\end{array}$ & Cyperus difformis & $\begin{array}{l}\text { Phyllanthus niruri, Phyllanthus } \\
\text { urinaria, Ludwigia parviflora, } \\
\text { Ammannia baccifera, Scoparia } \\
\text { dolcis, Chenopodium album }\end{array}$ \\
\hline $\mathrm{S}_{40} \mathrm{~F}_{40} \mathrm{~V}_{20}$ & 14 & $\begin{array}{l}\text { Brachiaria reptans. } \\
\text { Echinochloa colona, }\end{array}$ & - & - \\
\hline $\mathrm{S}_{40} \mathrm{~F}_{60} \mathrm{~V}_{0}$ & 15 & - & - & - \\
\hline $\mathrm{S}_{60} \mathrm{~F}_{0} \mathrm{~V}_{40}$ & 16 & $\begin{array}{l}\text { Brachiaria reptans. } \\
\text { Echinochloa colona, } \\
\text { Echinochloa crusgali, }\end{array}$ & $\begin{array}{l}\text { Cyperus difformis, } \\
\text { Cyperus rotundus }\end{array}$ & $\begin{array}{l}\text { Phyllanthus niruri, Ludwigia } \\
\text { parviflora, Scoparia dolcis, } \\
\text { Chenopodium album }\end{array}$ \\
\hline $\mathrm{S}_{60} \mathrm{~F}_{20} \mathrm{~V}_{20}$ & 17 & $\begin{array}{l}\text { Brachiaria reptans, } \\
\text { Echinochloa colona, } \\
\text { Echinochloa crusgali, }\end{array}$ & Cyperus difformis & $\begin{array}{l}\text { Phyllanthus niruri, Ludwigia } \\
\text { parviflora, Ammannia baccifera, } \\
\text { Scoparia dolcis, }\end{array}$ \\
\hline $\mathrm{S}_{60} \mathrm{~F}_{40} \mathrm{~V}_{0}$ & 18 & - & - & - \\
\hline $\mathrm{S}_{80} \mathrm{~F}_{0} \mathrm{~V}_{20}$ & 19 & $\begin{array}{l}\text { Cynodon dactylon, Brachiaria } \\
\text { reptans, Echinochloa colona, } \\
\text { Echinochloa crusgali, }\end{array}$ & Cyperus difformis & $\begin{array}{l}\text { Phyllanthus niruri, Ludwigia } \\
\text { parviflora, Ammannia baccifera, } \\
\text { Scoparia dolcis, Chenopodium } \\
\text { album }\end{array}$ \\
\hline $\mathrm{S}_{80} \mathrm{~F}_{20} \mathrm{~V}_{0}$ & 20 & $\begin{array}{c}\text { Brachiaria reptans, } \\
\text { Echinochloa crusgali, }\end{array}$ & - & - \\
\hline $\mathrm{S}_{100} \mathrm{~F}_{0} \mathrm{~V}_{0}$ & 21 & $\begin{array}{c}\text { Cynodon dactylon, Brachiaria } \\
\text { reptans, Echinochloa } \\
\text { crusgali, }\end{array}$ & Cyperus difformis & $\begin{array}{l}\text { Phyllanthus urinaria, Scoparia } \\
\text { dolcis, Chenopodium album }\end{array}$ \\
\hline
\end{tabular}


Fig.1 Number of broad leaf weeds $\mathrm{m}^{-2}$ observed in rice seedlings as influenced by different treatments

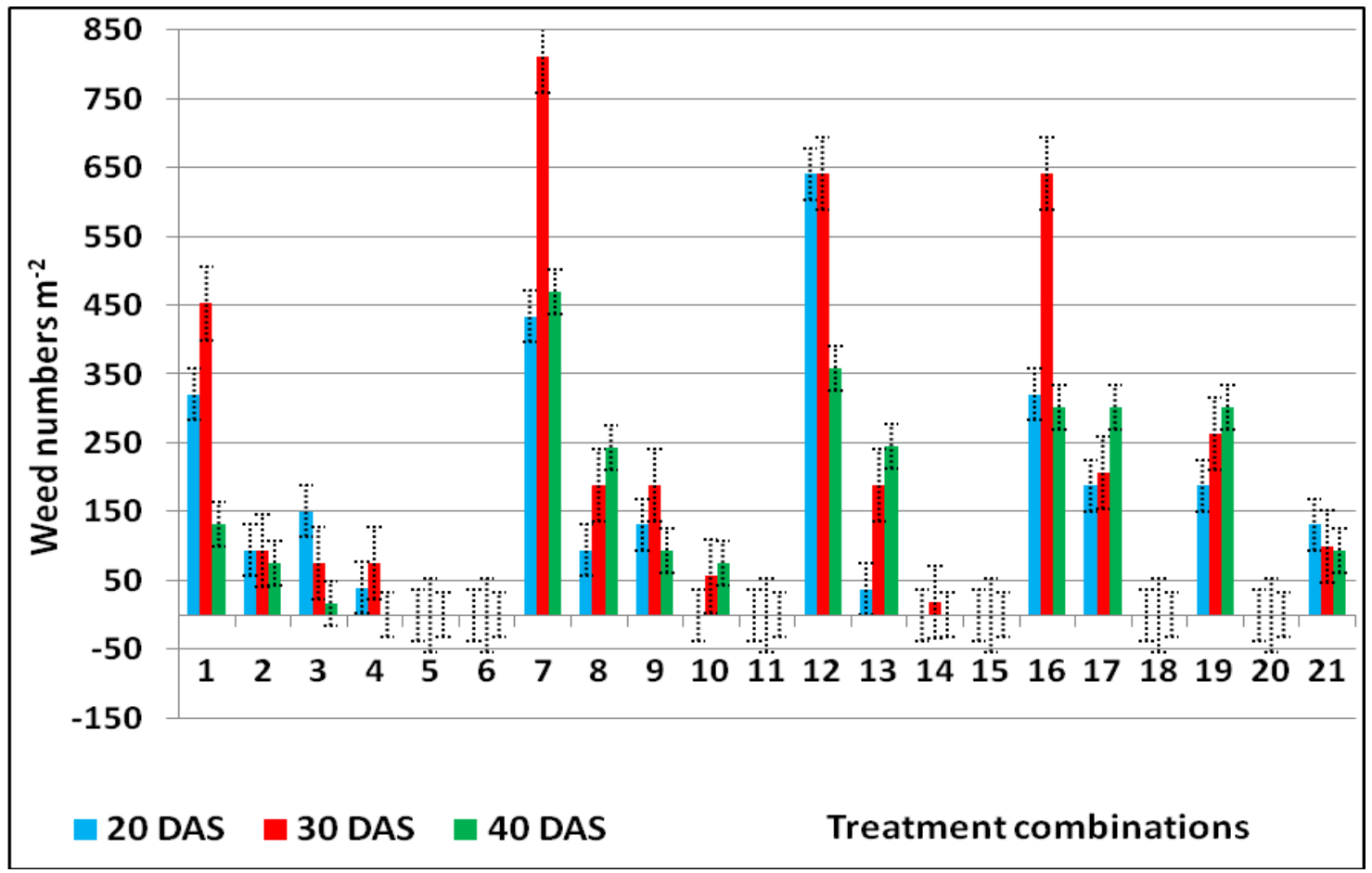

Fig.2 Number of grasses and sedges $\mathrm{m}^{-2}$ observed in rice seedlings as influenced by different treatments

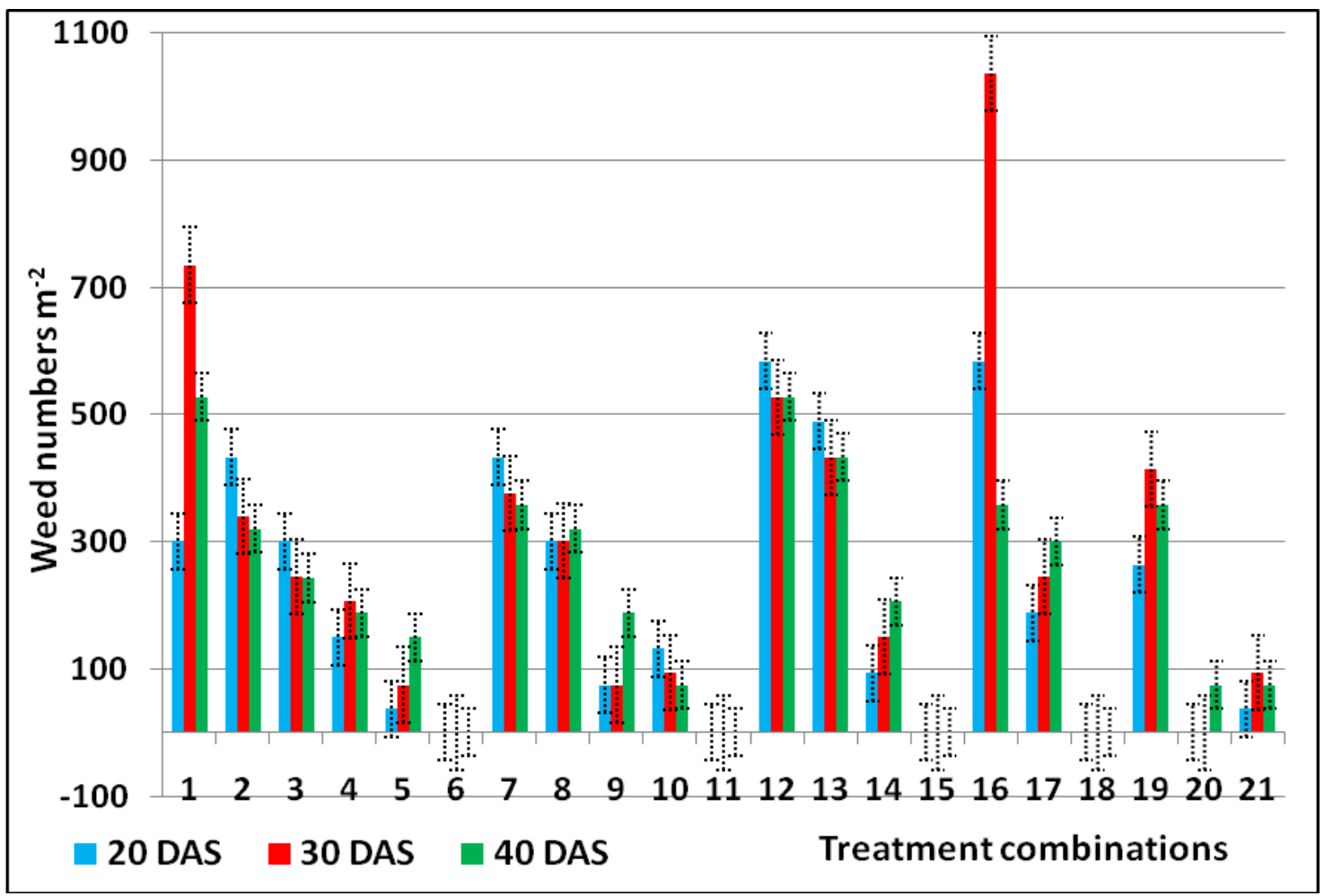


Fig.3 Total number of weeds $\mathrm{m}^{-2}$ observed in rice seedlings as influenced by different treatment combinations

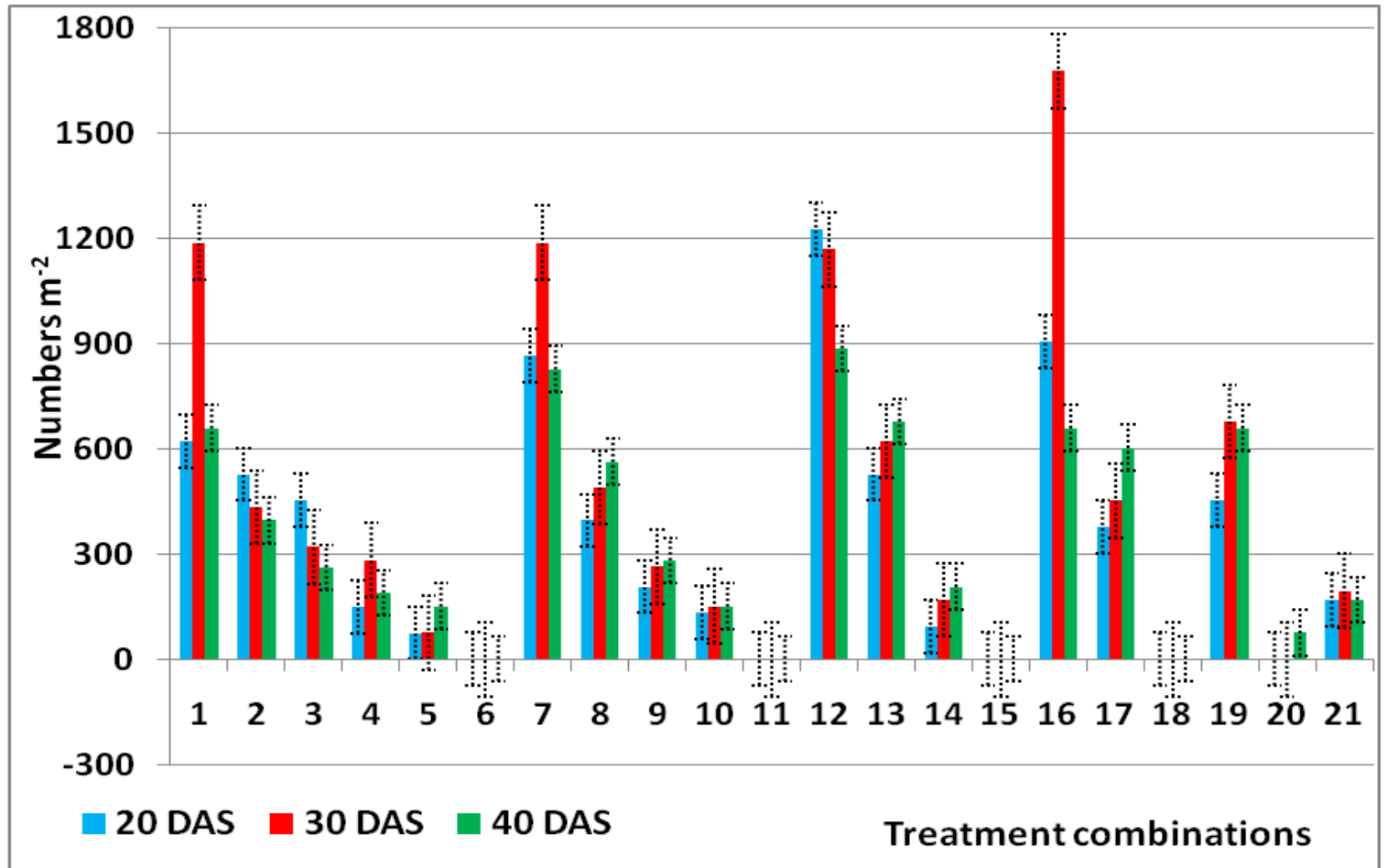

Fig.4 Total weed dry matter $\mathrm{m}^{-2}$ in rice seedlings as influenced by different treatment combinations

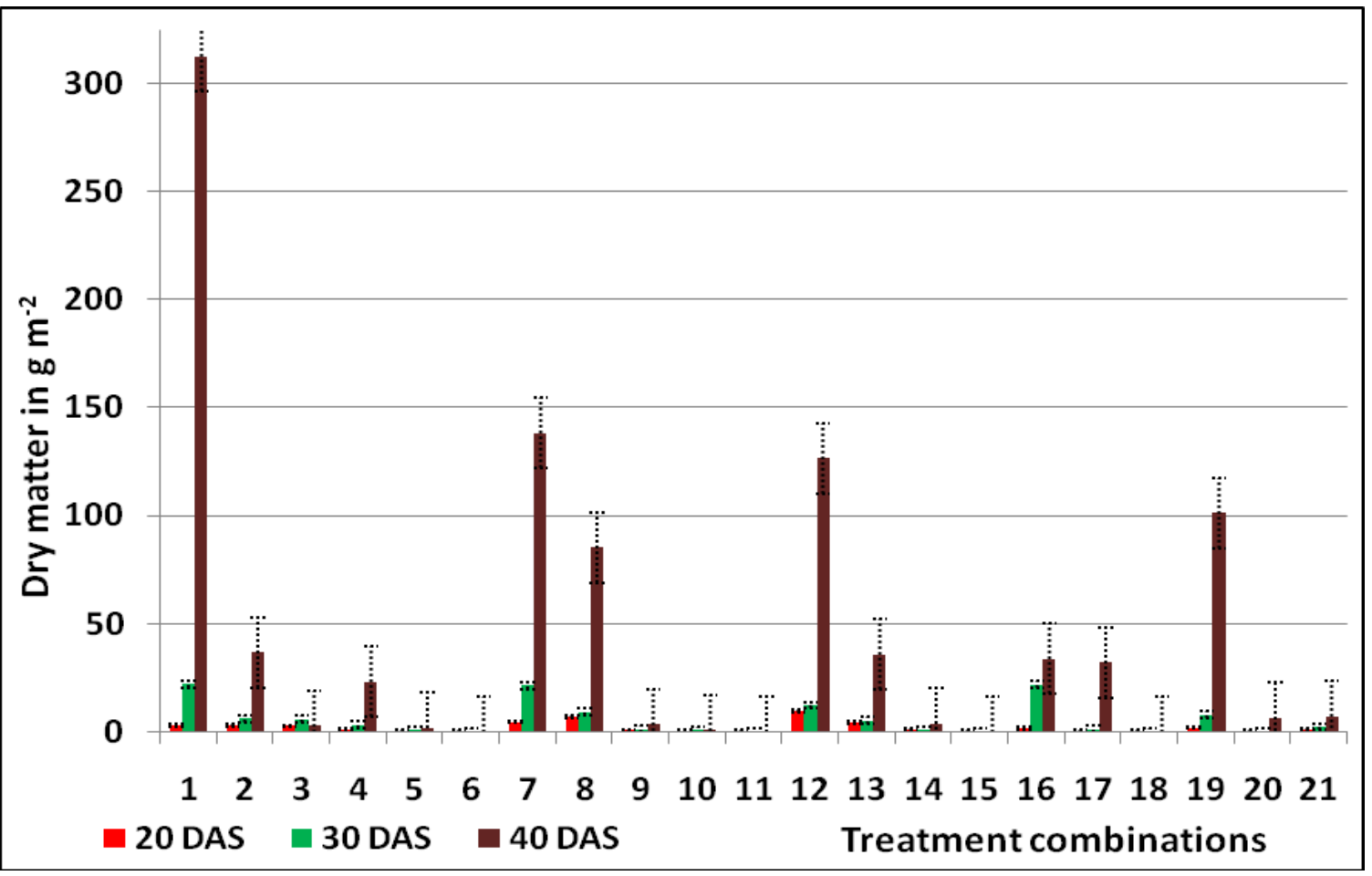


Plate.1 Floristic composition of weeds in experimental poly-bags

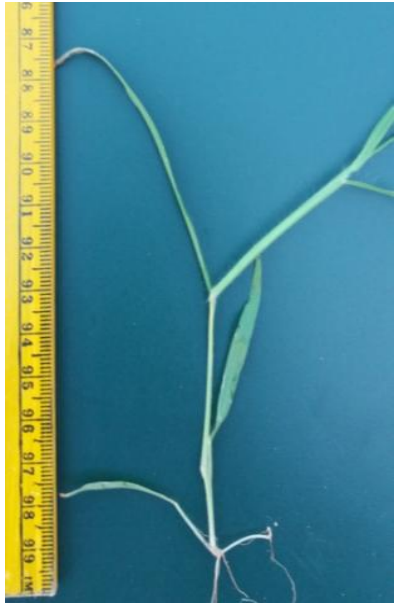

Bermuda grass (Cynodon dactylon $\mathrm{L}$.

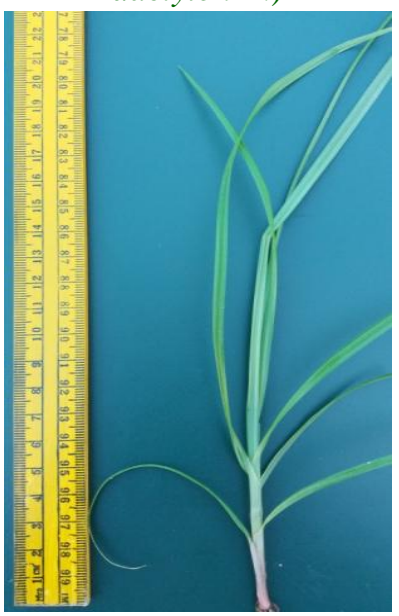

Flat sedge

(Cyperus difformis L.)

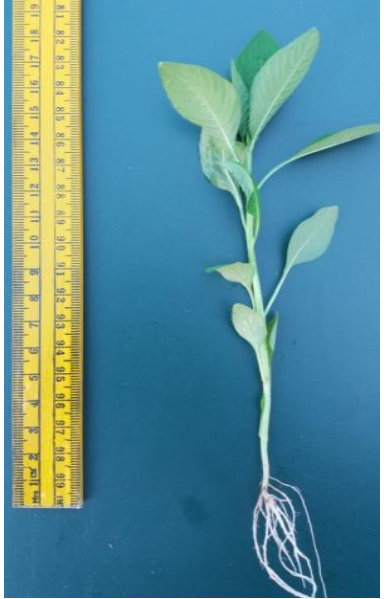

Creeping Water Primrose (Ludwigia parviflora Roxb.)

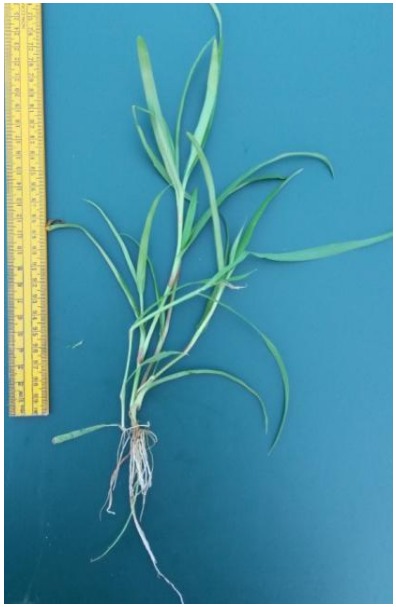

Running grass (Brachiaria reptans Gard. \& Hubb.)

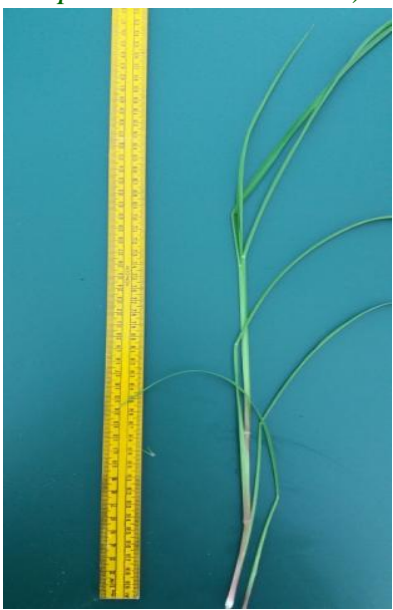

Nut sedge

(Cyperus rotundus L.)

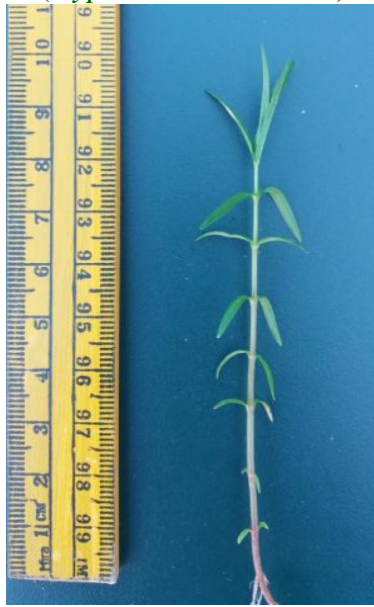

Monarch Red stem (Ammannia baccifera L.)

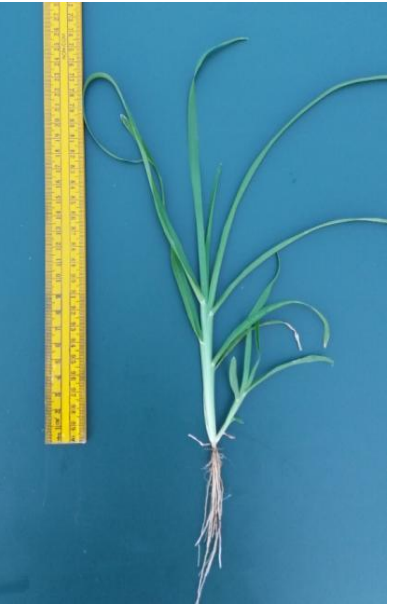

Jungle rice (Echinochloa colona $\mathrm{L}$.)

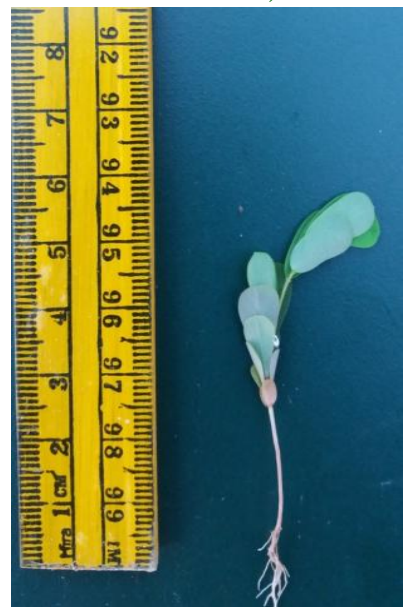

Stone breaker (Phyllanthus niruri L.)

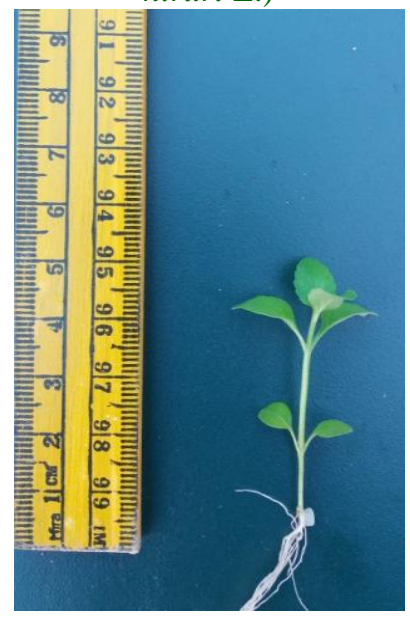

Goat weed (Scoparia dolcis L.)

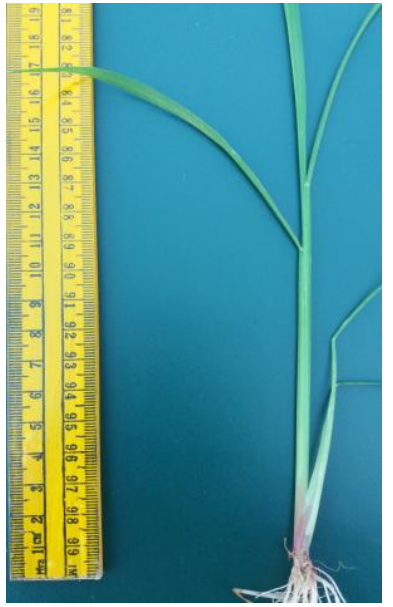

Barnyard grass (Echinochloa crusgali L.)

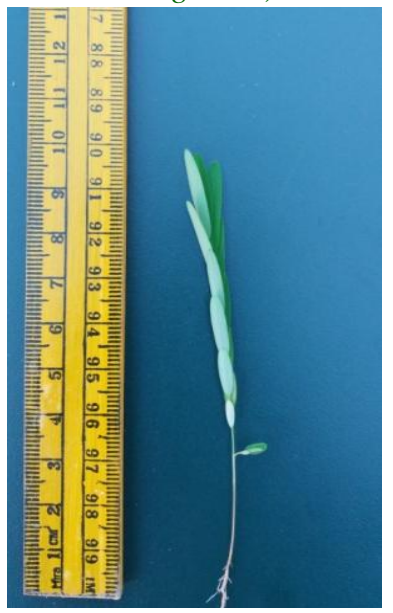

Chamber bitter (Phyllanthus urinaria $\mathrm{L}$.)

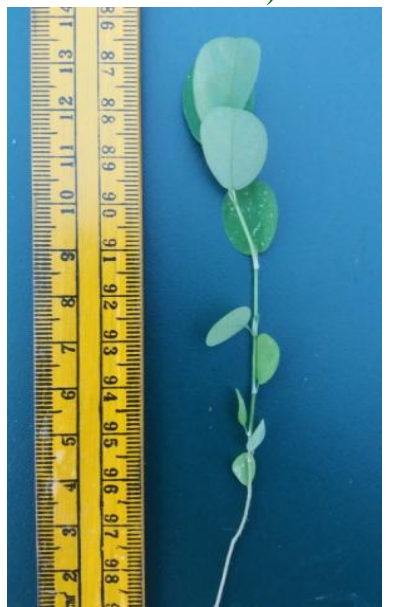

Goosefoot (Chenopodium album L.) 


\section{Dry matter of weeds}

The dry matter of weeds $\mathrm{m}^{-2}$ recorded at 20 , 30 and 40 DAS have been presented in Table 6 and depicted in Figure 4.

Similar to the total weed population, dry matter of weeds in $\mathrm{S}_{40} \mathrm{~F}_{0} \mathrm{~V}_{60}$ was the highest with significant difference from the rest treatment combinations at 20 DAS.

But, the weed dry matter in $\mathrm{S}_{0} \mathrm{~F}_{0} \mathrm{~V}_{100}$ surpassed it at 30 DAS $\left(22.004 \pm 1.540 \mathrm{~g} \mathrm{~m}^{-2}\right)$ and reached at its maximum at $40 \mathrm{DAS}$ $\left(312.808+34.409 \mathrm{~g} \mathrm{~m}^{-2}\right)$.

Apart from the weed free treatment combinations viz. $\quad \mathrm{S}_{0} \mathrm{~F}_{100} \mathrm{~V}_{0}, \quad \mathrm{~S}_{20} \mathrm{~F}_{80} \mathrm{~V}_{0}$, $\mathrm{S}_{40} \mathrm{~F}_{60} \mathrm{~V}_{0}$ and $\mathrm{S}_{60} \mathrm{~F}_{40} \mathrm{~V}_{0}$, the lowest dry matter of weeds was seen in $\mathrm{S}_{20} \mathrm{~F}_{60} \mathrm{~V}_{20}$ at 40 DAS.

Unlike rice seedling dry matter, the weed biomass followed a strict positive relationship with the levels of vermicompost that might be due to availability of plant nutrients in adequate quantities, in suitable proportions and also due to the ability of the weeds to preoccupy the rhizosphere well ahead of rice plants thereby suppressing the latter's growth rate.

\section{Floristic composition of weeds}

The floristic composition of different weed species of grasses, broadleaved and sedges in different treatment combinations at 40 DAS was studied. The common names and scientific names along with their photographs have been presented at Table 7 and Plate 1 . Cynodon dactylon was absent in absence of soil and Echinochloa sps was the ruling type. Brachiaria reptans was absent with 60 and 80 $\%$ substitution of vermicompost by fly ash. Cyperus difformis was more adaptable compared to Cyperus rotundus due to its wider existence in test-substrates. Among broadleaf weeds Phyllanthus sps. were abundantly present.

From the results so obtained it might be concluded that fly ash and vermicompost at varying levels in rice-nursery have their significant influences on the floristic composition of weeds. No broad leaf weed or grass or sedge could emerge in rice nursery in absence of vermicompost except in $100 \%$ soil with only very limited weed flora. The maximum number of broadleaf weeds emerged in soil with $80 \%$ vermicompost and the maximum number of grasses and sedges emerged in $100 \%$ vermicompost and also in soil with $60 \%$ vermicompost by weight. The highest dry matter of weeds was recorded in $100 \%$ vermicompost.

\section{Acknowledgements}

The technical and informative supports of the Department of Environment Science, Sambalpur University, Jyoti Vihar; the College of Agriculture, Chiplima and the Regional Research and Technology Transfer Station, Chiplima for conducting such waste to wealth making research in the fly-ash rich belt of Western Odisha are highly acknowledged.

\section{Conflict of interest}

There is no conflict of interest among the 5 authors.

\section{Ethical approval}

This article does not contain any studies with human participants or animals performed by any of the authors.

\section{References}

Central Electricity authority, Govt. of India. 2016. Retrieved 23 May 2016< 
https://en.wikipedia.org/wiki/List_of_po wer_stations_in_India>.

Gomez, K. A. and Gomez, A. A. 1984. Statistical procedures for agricultural research. Second edition. Wiley India, pp.200-206.

Parab, N., Mishra, S. and Bhonde, S. R. 2012. Prospects of bulk utilization of fly ash in agriculture for integrated nutrient management. Bulletin of National
Institute of Ecology, 23: 31-46. Rautaray, S. K., Ghosh, B. C., Mittra, B. N. 2003. Effect of fly ash, organic wastes and chemical fertilizers on yield, nutrient uptake, heavy metal content and residual fertility in a rice-mustard cropping sequence under acid lateritic soils. Bioresource Technology, 90: 275283.

\section{How to cite this article:}

Sanat Kumar Dwibedi, Sanjat Kumar Sahu, Ravi Kumar Patnaik, Ranjan Kumar Tarai and Abhiram Dash. 2017. Effects of Varying Levels of Fly Ash and Vermicompost Amendment on Floristic Composition of Weeds in Rice Nursery. Int.J.Curr.Microbiol.App.Sci. 6(12): 35653579. doi: https://doi.org/10.20546/ijcmas.2017.612.414 